\title{
FIRST PASSAGE TIMES FOR SYMMETRIC STABLE PROCESSES IN SPACE
}

\author{
BY \\ R. K. GETOOR(')
}

1. Introduction. Let $\{X(t) ; t \geqq 0\}$ be the symmetric stable process in $R^{N}$ of index $\alpha$ with $0<\alpha \leqq 2$; that is, a process with stationary independent increments, whose continuous transition density, $f(t, x-y)$, relative to Lebesgue measure in $R^{N}$ is uniquely determined by its Fourier transform

$$
e^{-t|\xi|^{\alpha}}=\int e^{i(x, \xi)} f(t, x) d x
$$

Here $\xi$ and $x$ are points in $R^{N}, d x$ is $N$-dimensional Lebesgue measure, $(x, \xi)$ is the usual inner product in $R^{N}$, and $|x|^{2}=(x, x)$. Throughout this paper integrals will be over all of $R^{N}$ unless explicitly indicated otherwise. If $r>0$ define $T(r)=\inf \{t:|X(t)|>r\}$. Thus $T(r)$ is the first passage time of the process $X(t)$ to exterior of the ball $\{x:|x|<r\}$. The main purpose of this paper is to prove the following formulas:

(A) $E_{x}\{T(r)\}=K(\alpha, N)\left(r^{2}-|x|^{2}\right)^{\alpha / 2}$,

(B) $E_{z}\left\{T(r)^{2}\right\}=\alpha r^{\alpha} K(\alpha, N)^{2} \int_{\left.|z|\right|^{2}}^{r^{2}}\left(t-|x|^{2}\right)^{\alpha / 2-1} F\left(-\frac{\alpha}{2}, \frac{N}{2} ; \frac{N+\alpha}{2} ; t r^{-2}\right) d t$

where

$$
K(\alpha, N)=\Gamma\left(\frac{N}{2}\right)\left[2^{\alpha} \Gamma\left(1+\frac{\alpha}{2}\right) \Gamma\left(\frac{N+\alpha}{2}\right)\right]^{-1} .
$$

These formulas hold provided $|x| \leqq r$. In (A) and (B) above $E_{x}\{\cdot\}$ denotes the expectation under the condition $X(0)=x$ and $F$ is the hypergeometric function. Note that

$$
\begin{aligned}
F\left(-\frac{\alpha}{2}, \frac{N}{2} ; \frac{N+\alpha}{2} ; t r^{-2}\right) \\
=\frac{\Gamma\left(\frac{N+\alpha}{2}\right)}{\Gamma\left(\frac{N}{2}\right) \Gamma\left(\frac{\alpha}{2}\right)} \int_{0}^{1} u^{N / 2-1}(1-u)^{\alpha / 2-1}\left(1-u t r^{-2}\right)^{\alpha / 2} d u .
\end{aligned}
$$

Presented to the Society, June 18, 1960; received by the editors July 13, 1960.

(1) This research was supported, in part, by the National Science Foundation. 
If $\alpha=2$ these results reduce easily to standard facts in the theory of Brownian motion (note that if $\alpha=2$ then $X(t / 2)$ is the usual Brownian motion). If $N=1$ and $\alpha=1$ formula (A) was obtained by Kac and Pollard [10]. More recently J. Elliot [4] has obtained (A) in the case $N=1$ and $0<\alpha<1$. We will prove these formulas when $0<\alpha<2$, the case $\alpha=2$ being well known and much simpler. Finally let us mention that formula (A) answers a conjecture of $\mathrm{Kac}[9]$ in the affirmative.

Formally it is easy to see that the functions $g(x)=E_{x}\{T(r)\}$ and $h(x)$ $=E_{x}\left\{T(r)^{2}\right\}$ satisfy the equations

$$
\begin{aligned}
\Lambda g & =-1, \\
\Lambda^{2} h & =2,
\end{aligned}
$$

where $\Lambda$ is the (infinitesimal) generator in an appropriate function space of the "absorbing barrier" process on $\{x:|x| \leqq r\}$. Our method consists in giving a precise meaning to (1.3) and then solving the resulting equations. In carrying out this procedure we obtain some results about the stable processes of interest in their own right. $\$ \$ 2,3$, and 4 contain the necessary preliminaries. Formula (A) is proved in $\$ 5$ and formula (B) in $\$ 6$. However, therather lengthy computations involved in the proof of $(B)$ are given in the appendix.

2. Preliminaries. Let $\{X(t) ; t \geqq 0\}$ be the symmetric stable process in $R^{N}$; that is, a process with stationary independent increments whose continuous transition density is given by (1.1). Of course, in order to specify $X(t)$ we must also give its initial distribution $\mu$. We always assume $\mu$ assigns mass one to some point $x$ in $R^{N}$ and write $P_{x}$ for the corresponding probability measure. It will be convenient to take as our basic probability space, $W$, all right continuous functions, $w$, from $0 \leqq t<\infty$ to $R^{N}$ which have left-hand limits everywhere and are bounded on bounded $t$-intervals. Our basic $\sigma$-algebra, $B$, is the usual $\sigma$-algebra generated by cylinder sets. Thus we have a collection of probability spaces $\left(W, B, P_{x}\right)$, one for each $x$ in $R^{N}$, such that $P_{x}\{X(0, w)=x\}$ $=1$. Here we have used the notation $X(t, w)=w(t)$.

Let $G$ be a bounded open subset of $R^{N}$ whose boundary has zero ( $N$ dimensional Lebesgue) measure. Let $\bar{G}$ be the closure of $G$ and define

$$
G(t)=\{w: X(\tau, w) \in \bar{G} \text { for all } \tau \text { with } 0 \leqq \tau \leqq t\} .
$$

It was shown in [7] that $G(t) \in B$. Note that we are using a slightly different notation from that of [7]. As in [7] we define

$$
K_{G}(t, x, A)=K(t, x, A)=P_{x}[\{X(t) \in A\} \cap G(t)] .
$$

Here $A$ is a Borel subset of $R^{N}$. Thus $K(t, x, A)$ is the probability of being in the set $A$ at time $t$ without ever having left $\bar{G}$ starting from $x$. It is clear that for each $(t, x)$ the measure $K(t, x, \cdot)$ is absolutely continuous with respect to Lebesgue measure and we denote the corresponding density by $k(t, x, y)$. Of 
course, $k(t, x, \cdot)$ is only determined almost everywhere. It was shown in [7] that $k$ could be chosen to be jointly measurable in its three variables.

Let $T=\inf \{t:|X(t)| \notin \bar{G}\}$. The right continuity of the paths implies that $T$ is a random variable. The fact that the process under consideration has the extended Markov property implies at once that the following first passage relation holds (this is often called the Désiré-André equation; see [12])

$$
f(t, x-y)=k(t, x, y)+\int_{0}^{t-} \int_{z \notin G} P_{x}[T \in d \tau, X(T) \in d z] f(t-\tau, z-y)
$$

for fixed $t>0, x$ in $G$ for almost all $y$ in $G$. We assume from now on that $k$ is chosen to satisfy (2.3) for all $t>0$ and $x, y$ in $G$. Using the properties of $P_{x}$ given in [7] it is easy to see that $k$ is jointly measurable in its variables. Also it is clear from $(2.3)$ that $k(t, x, y)$ is continuous in $y$ for fixed $t, x$. The following theorem is the basic result of this section.

Theorem 2.1. If $t>0$ is fixed, then $k(t, x, y)=k(t, y, x)$ for all $x, y$ in $G$.

Proof. We remark that it follows from the general results of [7] that $k(t, x, y)=k(t, y, x)$ almost everywhere on $G \times G$. However in the present paper we need this symmetry for all $x, y$ in order to establish the continuity of $k$ in its second variable.

Let $I_{t}$ be the characteristic function of the half open interval $0 \leqq \tau<t$. In the remainder of this proof $t>0, x, y$ in $G$ are fixed. If $H(x, y)$ denotes the second term on the right of (2.3) we have (we write $f(t, x, y)$ for $f(t, x-y)$ )

$$
B(x, y)=E_{x}\left\{f(t-T, X(T), y) I_{t}(T)\right\},
$$

and clearly it is enough to show that $H(x, y)=H(y, x)$. Consider the points $m t 2^{-n}$ for $m=0,1, \cdots$ and $n=1,2, \cdots$. Define

$$
T_{n}=\left\{\begin{array}{cl}
0 & \text { if } X(0) \notin \bar{G}, \\
m t 2^{-n} & \text { if } X\left(j t 2^{-n}\right) \in \bar{G} \text { for } j<m \text { and } X\left(m t 2^{-n}\right) \notin \bar{G}, \\
\infty & \text { if } X(0) \in \bar{G} \text { and there is no such } m .
\end{array}\right.
$$

Using the right continuity of the paths it is easy to see that $T_{n}$ decreases to $T$ and hence $X\left(T_{n}\right) \rightarrow X(T)$ as $n \rightarrow \infty$. Since $f(\tau, z, y)$ is a bounded continuous function of $\tau$ and $z$ for $0 \leqq \tau \leqq t$ and $z \notin G$ if $y$ is fixed in $G$, it follows that

$$
H_{n}(x, y)=E_{x}\left\{f\left(t-T_{n}, X\left(T_{n}\right), y\right) I_{t}\left(T_{n}\right)\right\} \rightarrow H(x, y)
$$

as $n \rightarrow \infty$. Thus it is sufficient to show that $H_{n}(x, y)=H_{n}(y, x)$.

Let $W_{0}$ be the subset of $W$ satisfying $X(0)=x$ and $X(t)=y$, i.e., the space of "tied paths." On the cylinder sets of $W_{0}$ of the form

$$
U=\left\{w: w\left(t_{j}\right) \in A_{j}, 0 \leqq t_{1}<t_{2}<\cdots<t_{p} \leqq t\right\}
$$

we define the finitely additive measure ${ }_{x} P_{y}$ as follows: 


$$
\begin{gathered}
{ }_{x} P_{y}(U)=f(t, x-y)^{-1} \int_{A_{1}} \ldots \int_{A_{p}} f\left(t_{1}, x-z_{1}\right) f\left(t_{2}-t_{1}, z_{2}-z_{1}\right) \\
\cdots f\left(t-t_{p}, y-z_{p}\right) d z_{p} \cdots d z_{1},
\end{gathered}
$$

where $f(0, x-y)=\delta(x-y)$. If we write out $H_{n}(x, y)$ we see at once that

$$
H_{n}(x, y)=f(t, x-y)_{x} P_{y}\left\{T_{n} \leqq t\right\} \text {. }
$$

Note that ${ }_{x} P_{y}\left\{T_{n}=t\right\}=0$ since $y$ is in $G$. Finally if we introduce $S_{n}=\max \left\{j t 2^{-n}: j \leqq 2^{n}, X\left(j t 2^{-n}\right) \notin \bar{G}\right\}$ or $S_{n}=t+1$ if there are no such $j$ and note that as subsets of $W_{0}$ we have

$$
\left\{T_{n} \leqq t\right\}=\bigcup_{m=1}^{2^{n}-1} \bigcup_{j=m}^{2^{n}-1}\left\{T_{n}=m t 2^{-n} ; S_{n}=j t 2^{-n}\right\} .
$$

it is not difficult to see that ${ }_{x} P_{y}\left\{T_{n} \leqq t\right\}={ }_{y} P_{x}\left\{T_{n} \leqq t\right\}$. This completes the proof of Theorem 2.1.

Finally the continuity of $k(t, x, y)$ in $y$ and Theorem 2.1 imply that $k(t, x, y)$ is continuous in $x$ on $G$ for fixed $t>0$ and $y$ in $G$.

3. The generator. It is well known $[7 ; 8]$ that the transformations

$$
\begin{aligned}
\left(S_{t} \phi\right)(x) & =\int f(t, x-y) \phi(y) d y \\
& =E_{x}\{\phi[X(t)]\}
\end{aligned}
$$

form a semi-group of self-adjoint contraction operators on $L_{2}\left(R^{N}\right)=L_{2}$ which is strongly continuous for $t \geqq 0$. Of course, we define $S_{0}=I$. If $\phi$ is in $L_{2}$ let $\hat{\phi}=F \phi$ be its $L_{2}$ Fourier transform, that is,

$$
\begin{aligned}
& \hat{\phi}(\xi)=(F \phi)(\xi)=\int e^{i(x, \xi)} \phi(x) d x, \\
& \phi(x)=(2 \pi)^{-N} \int \hat{\phi}(\xi) e^{-i(x, \xi)} d \xi .
\end{aligned}
$$

The integrals in (3.2) must, in general, be interpreted as $L_{2}$ integrals. Clearly

$$
\left(F S_{t} \phi\right)(\xi)=e^{-t|\xi|^{\alpha}} \hat{\phi}(\xi) .
$$

If $\Omega$ is the (infinitesimal) generator of the semi-group $\left\{S_{t} ; t \geqq 0\right\}$, then, as is easy to see [8, Theorem 21.4.2] we can characterize $\Omega$ as follows (of course $\left.D(\Omega) \subset L_{2}\right)$ :

$$
\begin{aligned}
D(\Omega) & =\left\{\phi:|\xi|^{\alpha} \hat{\phi}(\xi) \in L_{2}\right\}, \\
\Omega \phi & =F^{-1}\left[-|\xi|^{\alpha} \hat{\phi}(\xi)\right] \quad \text { if } \phi \in D(\Omega) .
\end{aligned}
$$

It was shown in [7] that the transformations 


$$
\begin{aligned}
\left(T_{t} \phi\right)(x) & =\int_{G} k(t, x, y) \phi(y) d y \\
& =E_{x}\{\phi[X(t)] ; X(\tau) \in \bar{G} \text { for all } \tau \text { with } 0 \leqq \tau \leqq t\}
\end{aligned}
$$

form a strongly continuous semi-group of self-adjoint contraction operators on $L_{2}(G)$. Note that $L_{2}(G)=L_{2}(\bar{G})$ since the boundary of $G$ is a null set. Let $\Lambda$ be the generator of $\left\{T_{t} ; t \geqq 0\right\}$; then $\Lambda$ is an unbounded self-adjoint operator in $L_{2}(G)$.

It will be convenient to introduce certain approximating semi-groups as in [7]. Let $V_{n}(x)=0$ for $x$ in $\bar{G}$ and $V_{n}(x)=n$ if $x$ not in $\bar{G}$. Define

$$
K^{(n)}(t, x, A)=E_{x}\left\{\exp \left(-\int_{0}^{t} V_{n}[X(\tau)] d \tau\right) ; X(t) \in A\right\}
$$

for $t>0, x$ in $R^{N}$, and $A$ a Borel subset of $R^{N}$. It was shown in [7] that $K^{(n)}(t, x, \cdot)$ has a density $k^{(n)}(t, x, y)$ with respect to Lebesgue measure that is jointly measurable in its variables. In addition it was shown that the operators

$$
\begin{aligned}
\left(T_{t}^{(n)} \phi\right)(x) & =\int k^{(n)}(t, x, y) \phi(y) d y \\
& =E_{x}\left\{\phi[X(t)] \exp \left(-\int_{0}^{t} V_{n}(X(\tau)) d \tau\right)\right\}
\end{aligned}
$$

form a strongly continuous semi-group of self-adjoint contraction operators on $L_{2}\left(R^{N}\right)$. Also $k^{(n)}(t, x, y) \leqq f(t, x-y)$ for almost all $y$ for fixed $(t, x)$. In the notation of [7] we have $S_{t}=T_{t}\left[0, R^{N}\right] ; T_{t}^{(n)}=T_{t}\left[V_{n}, R^{N}\right]$, and $T_{t}=T_{t}[0, G]$.

It will be necessary to consider these operators on $L_{1}$ as well as on $L_{2}$. It is immediate that $\left\{S_{t} ; t \geqq 0\right\}$ and $\left\{T_{t}^{(n)} ; t \geqq 0\right\}$ are semi-groups of contraction operators on $L_{1}\left(R^{N}\right)$, while $\left\{T_{t} ; t \geqq 0\right\}$ is a semi-group of contraction operators on $L_{1}(G)$. We use the same letter to denote the integral operator acting either on $L_{2}$ or $L_{1}$. It is well known [8, Theorem 23.13.1] that $\left\{S_{t} ; t \geqq 0\right\}$ is strongly continuous for $t \geqq 0$ on $L_{1}\left(R^{N}\right)$. Also it is easy to see that $\left\{T_{i}^{(n)} ; t \geqq 0\right\}$ and $\left\{T_{t} ; t \geqq 0\right\}$ are strongly continuous for $t \geqq 0$ on $L_{1}\left(R^{N}\right)$ and $L_{1}(G)$ respectively. Thus the generators of these semi-groups exist as densely defined closed operators in the appropriate spaces. Since domains are important in discussing these generators we will use a superscript " 1 " to denote the generator of a semi-group acting in an $L_{1}$ space. Thus $\Omega$ is the generator of $\left\{S_{t} ; t \geqq 0\right\}$ acting on $L_{2}\left(R^{N}\right)$ and $\Omega^{1}$ is the generator of $\left\{S_{t} ; t \geqq 0\right\}$ acting on $L_{1}\left(R^{N}\right)$. Similarly $\Lambda$ and $\Lambda^{1}$ are the generators of $\left\{T_{t} ; t \geqq 0\right\}$ acting on $L_{2}(G)$ and $L_{1}(G)$ respectively.

In the sequel it will be convenient to regard $L_{2}(G)$ as a closed subspace of $L_{2}\left(R^{N}\right)$ by setting $\phi(x)=0$ for $x$ not in $G$ if $\phi$ is in $L_{2}(G)$. Note that multiplication by $I_{G}$, the characterististic function of $G$, is the orthogonal projec- 
tion from $L_{2}\left(R^{N}\right)$ onto $L_{2}(G)$. Similarly we will regard $L_{1}(G)$ as a closed subspace of $L_{1}\left(R^{N}\right)$. The following approximation lemma is now easily proved; for example, by methods analogous to those used in the proof of Theorem 4.1 of $[7]$.

LEMMA 3.1. (i) If $\phi$ is in $L_{2}\left(R^{N}\right)$ then $T_{t}^{(n)} \phi \rightarrow T_{i} I_{Q} \phi$ weakly as $n \rightarrow \infty$.

(ii) If $\phi$ is in $L_{1}\left(R^{N}\right)$ and $\psi$ is a bounded measurable function with compact support, then

$$
\int T_{t}^{(n)} \phi \cdot(x) \psi(x) d x \rightarrow \int T_{t} I_{G \phi} \cdot(x) \psi(x) d x \quad \text { as } n \rightarrow \infty .
$$

In $\left[6\right.$, Theorem 5.1] it was shown that the generator of $\left\{T_{t}^{(n)} ; t \geqq 0\right\}$ on $L_{2}\left(R^{N}\right)$ is $\Omega-V_{n}$ using only Banach space methods. Thus with only trivial modifications of the argument given there we find that the generator of $\left\{T_{t}^{(n)} ; t \geqq 0\right\}$ on $L_{1}\left(R^{N}\right)$ is $\Omega^{1}-V_{n}$. We now state the main theorem of this section.

THEOREM 3.1. (i) If $\phi$ is in $D(\Omega)$ and $I_{G} \phi=\phi$, then $\phi$ is in $D(\Lambda)$ and $\Lambda \phi=I_{G} \Omega \phi$.

(ii) If $\phi$ is in $D\left(\Omega^{1}\right)$ and $I_{G} \phi=\phi$ then $\phi$ is in $D\left(\Lambda^{1}\right)$ and $\Lambda^{1} \phi=I_{G} \Omega^{1} \phi$.

Proof. Since the proofs of these two statements are similar, we will confine our attention to (ii). Let $I_{\lambda}, J_{\lambda}^{(n)}$, and $J_{\lambda}$ be the resolvents of the semigroups $\left\{S_{t} ; t \geqq 0\right\},\left\{T_{t}^{(n)} ; t \geqq 0\right\}$, and $\left\{T_{t} ; t \geqq 0\right\}$ respectively. Let $\lambda>0$ be fixed throughout this proof. Now if $\phi$ is in $D\left(\Omega^{1}\right)$ then $\phi$ is in $D\left(\Omega^{1}-V_{n}\right)$ and hence $\phi=J_{\lambda}^{(n)} \psi_{n}$ for some $\psi_{n}$ in $L_{1}\left(R^{N}\right)$. But $V_{n} \phi=0$ since $\phi$ is in $L_{1}(G)$. Therefore

$$
\psi_{n}=\left[\lambda-\left(\Omega^{1}-V_{n}\right)\right] \phi=\left(\lambda-\Omega^{1}\right) \phi .
$$

Defining $\psi=\left(\lambda-\Omega^{1}\right) \phi$ we see that $J_{\lambda}^{(n)} \psi_{n}=J_{\lambda}^{(n)} \psi$. If $b$ is a bounded measurable function with compact support, then using Lemma 3.1 (ii) it follows readily that $\langle b, \phi\rangle=\left\langle b, J_{\lambda}^{(n)} \psi_{n}\right\rangle=\left\langle b, J_{\lambda}^{(n)} \psi\right\rangle \rightarrow\left\langle b, J_{\lambda} I_{G} \psi\right\rangle$. Here $\langle b, h\rangle=\int b(x) h(x) d x$ for any $b$ in $L_{\infty}$ and $h$ in $L_{1}$. Thus $\langle b, \phi\rangle=\left\langle b, J_{\lambda} I_{a} \psi\right\rangle$ for all bounded measurable functions $b$ with compact support, and therefore $\phi=J_{\lambda} I_{G} \psi$. But this implies that $\phi$ is in $D\left(\Lambda^{1}\right)$ and $\left(\lambda-\Lambda^{1}\right) \phi=I_{G} \psi=I_{G}\left(\lambda-\Omega^{1}\right) \phi$, or equivalently, $\Lambda^{1} \phi$ $=I_{G} \Omega^{1} \phi$ since $I_{G} \phi=\phi$. This completes the proof of Theorem 3.1.

4. The inverse of the generator. Throughout this section, as in the previous sections, $G$ denotes a bounded open subset of $R^{N}$ whose boundary has zero measure. In $\S 2$ we showed that $k(t, x, y)$ is a continuous function of $x$ for fixed $t$ and $y$. Also we have the following estimate

$$
k(t, x, y) \leqq f(t, x-y) \leqq c t^{-N / \alpha}
$$

where $c$ is a positive constant independent of $x$ and $y$. In this section we prove only what will be needed in $\S 5$. The results that we obtain certainly are not the most general possible along these lines. 
THEOREM 4.1. If $\phi$ is a bounded measurable function on $G$, then $\int_{0}^{\infty} d t \int_{G} k(t, x, y) \phi(y) d y$ exists for all $x$ in $G$ and defines a bounded continuous function on $G$.

Proof. Since $\phi$ is bounded it is obvious that $\int_{0}^{1} d t \int a k(t, x, y) \phi(y) d y$ exists for all $x$ in $G$, and if $\alpha<N$ then (4.1) implies the existence of the integral over $(1, \infty)$. Moreover each of these estimates is uniform in $x$. Since $\int a k(t, x, y) \phi(y) d y$ is clearly a continuous function on $G$ for each fixed $t>0$, Theorem 4.1 follows in the case $\alpha<N$.

To treat the case $N=1 \leqq \alpha<2$ we use the following argument. In $\$ 6$ of [7] it was shown that

$$
k(t, x, y)=\sum_{j=1}^{\infty} e^{-\lambda_{j} t} \phi_{j}(x) \phi_{j}(y)
$$

where $\left\{-\lambda_{j}\right\}$ is the discrete spectrum of $\Lambda$, the generator of $\left\{T_{t} ; t \geqq 0\right\}$ acting on $L_{2}(G)$, and $\left\{\phi_{j}\right\}$ the corresponding complete orthonormal system in $L_{2}(G)$. Also $0 \leqq \lambda_{1} \leqq \lambda_{2} \leqq \cdots$, , and each $\lambda_{j}$ is repeated according to its finite multiplicity. It was shown in [7] that eigenfunctions $\phi_{j}$ may be chosen to satisfy

$$
e^{-\lambda_{j} t} \phi_{j}(x)=\int_{G} k(t, x, y) \phi_{j}(y) d y
$$

for all $x$ in $G$ and $\iota>0$. Since $k(t, x, y)$ is continuous in $x$ for fixed $t, y$, it follows that each $\phi_{j}$ is continuous on $G$. Moreover $\lambda_{1}>0$, since if $\phi$ were an eigenfunction corresponding to the eigenvalue 0 we would have, letting $\psi=|\phi|$,

$$
\psi(x) \leqq \int_{G} f(t, x-y) \psi(y) d y \leqq K \int e^{-t|\xi|^{\alpha}} \psi(\xi) d \xi \rightarrow 0 \quad \text { as } t \rightarrow \infty .
$$

Thus $\lambda=0$ is not an eigenvalue. Finally the sum in (4.2) converges absolutely for $t>0, x, y$ in $G$. The above remarks hold for all values of $N$ and $\alpha$.

Returning to the case $N=1 \leqq \alpha<2$ it follows from the results of [2, Equation (3.6)] that $\lambda_{j} \sim M j^{\alpha}$ as $j \rightarrow \infty$ where $M$ is a constant independent of $j$. Straightforward estimates yield

$$
\int_{G} d x \int_{1}^{\infty} t d t \int_{G} k(t, x, y)|\phi(y)| d y \leqq K \sum_{j=1}^{\infty} \lambda_{j}^{-2}<\infty,
$$

and therefore using the Fubini theorem the integral in Theorem 4.1 exists for almost all $x$ in $G$.

Since $G$ is bounded it is contained in some ball (interval since $N=1$ ) $\left\{x:|x|<R_{0}\right\}$. Choose $R>3 R_{0}$ and let $k^{R}(t, x, y)$ be the transition density for the absorbing barrier process on $B=\{x:|x|<R\}$. Applying what has just been proved to $B$ there exists an $x_{0}$ in $G$ such that $\int_{0}^{\infty} d t \int_{B} k^{R}\left(t, x_{0}, y\right) d y<\infty$. Since $X(t)$ has independent increments it is not difficult to see that for each 
fixed $x$ in $G$ we have $k(t, x, y) \leqq k^{R}\left(t, x_{0}, y+x_{0}-x\right)$ for almost all $y$ in $G$. Thus for each fixed $x$ in $G$

$$
\int_{0}^{\infty} d t \int_{G} k(t, x, y) d y \leqq \int_{0}^{\infty} d t \int_{B} k\left(t, x_{0}, y\right) d y<\infty,
$$

and since this estimate is uniform in $x$, Theorem 4.1 follows in the case $N=1 \leqq \alpha<2$.

Recalling that $\Lambda^{1}$ is the generator of $\left\{T_{t} ; t \geqq 0\right\}$ acting on $L_{1}(G)$, we state the following theorem. then

TheOREM 4.2. Suppose $\psi$ is in $D\left(\Lambda^{1}\right)$ and let $\phi=\Lambda \psi$. If $\phi$ is bounded on $G$,

$$
\int_{0}^{\infty} d t \int_{a} k(t, x, y) \phi(y) d y=-\psi(x)
$$

almost everywhere on $G$.

Proof. Using (4.1) and the fact that each $T_{t}$ is a contraction on $L_{1}(G)$ it is not difficult to see that $T_{\mathrm{t} g} \rightarrow 0$ in $L_{1}(G)$ as $t \rightarrow \infty$ for all $g$ in $L_{1}(G)$. Thus

$$
\int_{0}^{\infty} T_{t} \phi d t=\int_{0}^{\infty} T_{t} \Lambda^{1} \psi d t=\int_{0}^{\infty} \frac{d}{d t}\left(T_{t} \psi\right) d t=\left.T_{t} \psi\right|_{0} ^{\infty}=-\psi,
$$

where we have used the strong continuity of $\left\{T_{t} ; t \geqq 0\right\}$ and the above remark. The above integrals are $L_{1}(G)$ Bochner (actually Riemann) integrals. Theorem 4.2 is now immediate since the $L_{1}(G)$ integral must agree almost everywhere with the pointwise integral whose existence was established in Theorem 4.1.

5. Proof of formula (A). In this section we assume that $G=\{x:|x|<1\}$. As in the introduction we define $T=T(1)=\inf \{t:|X(t)|>1\}$. It follows easily from our definitions that

$$
P_{x}[T>t]=\int_{a} k(t, x, y) d y .
$$

TheOREM 5.1. If $|x|<1$, then

$$
E_{x}(T)=\int_{0}^{\infty} d t \int_{|y|<1} k(t, x, y) d y .
$$

Proof. Let $F_{x}(t)=P_{x}[T>t]$, then

$$
E_{x}(T)=-\int_{0}^{\infty} t d F_{x}(t)=-\lim _{t \rightarrow \infty} t F_{x}(t)+\int_{0}^{\infty} F_{x}(t) d t
$$

Using (5.1) and Theorem 4.1 this last integral exists for all $x$ with $|x|<1$. On the other hand 


$$
F_{x}(t)=\sum_{j=1}^{\infty} \sigma^{-\lambda} t \phi_{j}(x)\left(1, \phi_{j}\right)
$$

where the sum converges absolutely for fixed $t$ and $x$. If $t_{0}>\lambda_{1}^{-1}$, then $t e^{-\lambda_{f} t}$ $\leqq t_{0} e^{-\lambda_{j} t_{0}}$ for all $j$ and $t>t_{0}$. Thus by the bounded convergence theorem $t F_{x}(t) \rightarrow 0$ as $t \rightarrow \infty$. This establishes Theorem 5.1.

Corollary 5.1. Suppose $g$ is in $D\left(\Lambda^{1}\right)$ and $g$ is continuous on $\{x:|x|<1\}$. If $\Lambda^{1} g=-1$, then $E_{x}(T)=g(x)$ provided $|x|<1$.

Proof. Combining Theorem 4.2 and 5.1 we see that $E_{x}(T)=g(x)$ almost everywhere on $G$. But Theorems 4.1 and 5.1 imply that $E_{x}(T)$ is a continuous function of $x$ on $G$ and since $g$ is continuous by hypothesis, we obtain the desired equality for all $x$ in $G$.

It is known (Theorem 21.4.2 of [8]; see also page 658 of [8]) that the generator $\Omega^{1}$ of $\left\{S_{t} ; t \geqq 0\right\}$ on $L_{1}\left(R^{N}\right)$ may be described as follows:

(i) $D\left(\Omega^{1}\right)=\left\{\phi: \phi \in L_{1}\left(R^{N}\right)\right.$ and $|\xi|^{\alpha} \hat{\phi}(\xi)$ is the Fourier transform of some element in $\left.L_{1}\left(R^{N}\right)\right\}$.

(ii) If $\phi \in D\left(\Omega^{1}\right)$, then $\Omega^{1} \phi$ is the element in $L_{1}\left(R^{N}\right)$ whose Fourier transform is $-|\xi|^{\alpha} \hat{\phi}(\xi)$.

In [8] this is proved in the case $N=1$, but the extension to general $N$ is straightforward. Thus in view of Theorem 3.1 to solve the equation $\Lambda^{1} g=-1$ it would be sufficient to find a $g$ in $D\left(\Omega^{1}\right)$ which is zero for $|x|>1$ and such that $\Omega^{1} g=-1$ for $|x|<1$. It is clear from the probabilistic considerations that $g$ should be a radial function and its Fourier transform $g(|\xi|)$ should also be radial. Using the Fourier inversion formula for radial functions [3, p. 69] the above conditions become

$$
\begin{aligned}
g(x)=(2 \pi)^{-N / 2}|x|^{1-N / 2} \int_{0}^{\infty} t^{N / 2} g(t) J_{(N-2) / 2}(t|x|) d t & =0 \\
& \text { if }|x|>1 \\
-\left(\Omega^{1} g\right)(x)=(2 \pi)^{-N / 2}|x|^{1-N / 2} \int_{0}^{\infty} t^{\alpha+N / 2} g(t) J_{(N-2) / 2}(t|x|) d t=1 & \text { if }|x|<1 .
\end{aligned}
$$

These equations are "dual" integral equations of the type considered by Titchmarsh $[13$, p. 337$]$ and one can write down the solution $g$ at least formally. It is then possible to invert $\hat{g}$, again formally, to obtain $g$. Unfortunately it seems difficult to justify these formal calculations and so we will write down the $g$ obtained formally in this manner and then verify directly that it is in $D\left(\Lambda^{1}\right)$ and satisfies $\Lambda^{1} g=-1$.

TheOREM 5.2. Let $g(x)=K(\alpha, N)\left(1-|x|^{2}\right)^{\alpha / 2}$ if $|x|<1$ and $g(x)=0$ if $|x| \geqq 1$ where $K(\alpha, N)$ is given by (1.2); then $g \in D\left(\Omega^{1}\right)$ provided $0<\alpha<2$. If 


$$
\begin{aligned}
H(x)=1 & \text { if }|x|<1, \\
H(x)= & {\left[\Gamma\left(-\frac{\alpha}{2}\right) \Gamma\left(\frac{N+\alpha+2}{2}\right)\right]^{-1} \Gamma\left(\frac{N}{2}\right)|x|^{-N-\alpha} } \\
& \cdot F\left(\frac{\alpha+2}{2}, \frac{N+\alpha}{2} ; \frac{N+\alpha+2}{2} ;|x|^{-2}\right) \\
& \text { if }|x|>1,
\end{aligned}
$$

then $\Omega^{1} g=-H$.

Proof. In view of the characterization of $\Omega^{1}$ we must show that $H$ is in $L_{1}$ and $\hat{H}(\xi)=|\xi| \alpha g(\xi)$. Clearly $H$ is integrable provided we stay away from the sphere $|x|=1$. Using the transformation $[11$, p. 9]

$$
F(a, b ; c ; 8)=(1-z)^{c-a-b} F(c-a, c-b ; c ; z)
$$

provided $|z|<1$ and the fact that the power series for $F(a, b ; c ; z)$ converges absolutely on $|z|=1$ if $a+b-c<0$, we see that $\left.\left.H(x) \sim K|1-| x\right|^{2}\right|^{-\alpha / 2}$ as $|x| \downarrow 1$. Since $0<\alpha<2$ this implies that $H$ is in $L_{1}$.

It is an immediate consequence of formula $33, \$ 8.5$ of [5] that

$$
\begin{aligned}
\hat{g}(\xi) & =(2 \pi)^{N / 2} K(\alpha, N)|\xi|^{1-N / 2} \int_{0}^{1} t^{N / 2}\left(1-t^{2}\right)^{\alpha / 2} J_{(N-2) / 2}(t|\xi|) d t \\
& =(2 \pi)^{N / 2} \Gamma\left(\frac{N}{2}\right)\left[2^{\alpha / 2} \Gamma\left(\frac{N+\alpha}{2}\right)|\xi|^{(N+\alpha) / 2}\right]^{-1} J_{(N+\alpha) / 2}(|\xi|) .
\end{aligned}
$$

We must now compute $\hat{H}(\xi)=\phi(\xi)+\psi(\xi)$ where

$$
\begin{aligned}
\phi(\xi) & =(2 \pi)^{N / 2}|\xi|^{1-N / 2} \int_{0}^{1} t^{N / 2} J_{(N-2) / 2}(t|\xi|) d t \\
& =(2 \pi)^{N / 2}|\xi|^{-N / 2} J_{N / 2}(|\xi|),
\end{aligned}
$$

and

$$
\begin{aligned}
\psi(\xi)= & {\left[\Gamma\left(-\frac{\alpha}{2}\right) \Gamma\left(\frac{N+\alpha+2}{2}\right)\right]^{-1}(2 \pi)^{N / 2} \Gamma\left(\frac{N}{2}\right)|\xi|^{1-N / 2} } \\
& \times \int_{1}^{\infty} t^{-N / 2-\alpha} F\left(\frac{\alpha+2}{2}, \frac{N+\alpha}{2} ; \frac{N+\alpha+2}{2} ; t^{-2}\right) J_{(N-2) / 2}(t|\xi|) d t .
\end{aligned}
$$

Calling the integral $I_{1}$ and integrating by parts using the first differentiation formula on p. 10 of [11], we find 


$$
\begin{aligned}
I_{1}= & \frac{\Gamma\left(\frac{N+\alpha+2}{2}\right) \Gamma(1-\alpha / 2)}{\alpha \Gamma\left(\frac{N+2}{2}\right)} J_{(N-2) / 2}(|\xi|) \\
& -\frac{|\xi|}{\alpha} \int_{1}^{\infty} t^{1-\alpha-N / 2} F\left(\frac{\alpha}{2}, \frac{N+\alpha}{2} ; \frac{N+\alpha+2}{2} ; t^{-2}\right) J_{N / 2}(|\xi| t) d t .
\end{aligned}
$$

This last integral, $I_{2}$, is conditionally convergent and integrating by parts again using this time the third differentiation formula on p. 10 of [11] we obtain

$$
\begin{aligned}
I_{2}= & -|\xi|^{-1} J_{(N+2) / 2}(|\xi|) \frac{\Gamma((N+\alpha+2) / 2) \Gamma(1-\alpha / 2)}{\Gamma((N+2) / 2)} \\
& +\frac{N+\alpha}{|\xi|} \int_{1}^{\infty} t^{-N / 2}\left(t^{2}-1\right)^{-\alpha / 2} J_{(N+2) / 2}(|\xi| t) d t .
\end{aligned}
$$

This last integral, $I_{3}$, is easily evaluated (see formula 32, $\$ 8.5$ of [5]) yielding

$$
I_{3}=2^{-\alpha / 2} \Gamma(1-\alpha / 2)|\xi|^{\alpha / 2-1} J_{(N+\alpha) / 2}(|\xi|) .
$$

Combining these formulas and using the recursion formula for Bessel functions it is not difficult to see that $\hat{H}(\xi)=|\xi| \alpha \xi(\xi)$. This completes the proof of Theorem 5.2.

If we now take into account Corollary 5.1 and Theorems 3.1 and 5.2 we find immediately that

$$
E_{x}(T)=K(\alpha, N)\left(1-|x|^{2}\right)^{\alpha / 2}
$$

provided $0<\alpha<2$ and $|x|<1$. However (5.5) also holds when $|x|=1$ since both sides are zero then. But (5.5) is formula (A) in the special case $r=1$. formula (A) for arbitrary $r>0$ now follows easily from (5.5) and the fact that

$$
P_{x}[r X(t) \in B]=P_{r z}\left[X\left(r^{\alpha} t\right) \in B\right]
$$

for all $r>0$ and all Borel subsets $B$ of $R^{N}$. We omit the details.

6. Formula (B). In this section, as in $\$ 5$, let $T=T(1)=\inf \{t:|X(t)|>1\}$ and $G=\{x:|x|<1\}$. Exactly as in $\$ 5$ we find that for $|x|<1$ we have

$$
\begin{aligned}
E_{x}\left(T^{2}\right) & =\int_{0}^{\infty} t^{2} d F_{x}(t) \\
& =2 \int_{0}^{\infty} t d t \int_{a} k(t, x, y) d y .
\end{aligned}
$$

Also arguments similar to those used in Theorems 4.1 and 4.2 show that if $\psi$ is in $D\left(\left(\Lambda^{1}\right)^{2}\right)$ and $\phi=\left(\Lambda^{1}\right)^{2} \psi$ is bounded then $\int_{0}^{\infty} t d t \int_{G} k(t, x, y) \phi(y) d y=\psi(x)$ 
almost everywhere on $G$ and that the integral is a continuous function of $x$ on $G$. Thus we have the following theorem.

THEOREM 6.1. Suppose $h$ is $D\left(\left(\Lambda^{1}\right)^{2}\right)$ and is continuous on $G$, then if $\left(\Lambda^{1}\right)^{2} h=2$ we have $E_{x}\left(T^{2}\right)=h(x)$ for $|x|<1$.

We know from $\delta 5$ that if $g(x)=K(\alpha, N)\left(1-|x|^{2}\right)^{\alpha / 2}$ then $\Lambda^{1} g=-1$. Therefore if we find an $h$ such that

$$
\Lambda^{1} h=-2 g
$$

it will follow that $\left(\Lambda^{1}\right)^{2} h=2$. To solve (6.2) we proceed as in 85 . We look for an $h$ such that $h$ is in $D\left(\Omega^{1}\right), h=0$ for $|x|>1$, and $\Omega^{1} h=-2 g$ for $|x|<1$. This again leads to a pair of dual integral equations for $h$ that we can solve formally. In turn we obtain $h$ formally by inversion. It is then necessary to verify directly that $h$ is the desired solution. The necessary calculations are contained in the following theorem.

Theorem 6.2. Suppose $0<\alpha<2$ and let

$$
h(x)=\alpha K(\alpha, N)^{2} \int_{|x|^{2}}^{1}\left(t-|x|^{2}\right)^{\alpha / 2-1} F\left(-\frac{\alpha}{2}, \frac{N}{2} ; \frac{N+\alpha}{2} ; t\right) d t
$$

if $|x|<1$ and $h(x)=0$ if $|x| \geqq 1$, then $h \in D\left(\Omega^{1}\right)$. In fact, if

$$
\begin{aligned}
& q(x)=2 g(x) \\
& \text { if }|x|<1 \text {, } \\
& q(x)=2 K(\alpha, N) \Gamma\left(\frac{N}{2}\right)\left[\Gamma\left(-\frac{\alpha}{2}\right) \Gamma\left(\frac{N+\alpha}{2}\right)|x|^{N+\alpha}\right]^{-1} \\
& \times \int_{0}^{1} t^{(N+\alpha-2) / 2}\left(1-t /|x|^{2}\right)^{-1-\alpha / 2} F\left(-\frac{\alpha}{2}, \frac{N}{2} ; \frac{N+\alpha}{2} ; t\right) d t \\
& \text { if }|x|>1 \text {, }
\end{aligned}
$$

then $q$ is in $L_{1}$ and $|\xi|{ }^{\alpha} h(\xi)=\hat{q}(\xi)$, i.e., $\Omega^{1} h=-q$.

The proof of this theorem involves a rather lengthy computation and will therefore be given in the appendix. It is now immediate that $\left(\Lambda^{1}\right)^{2} h=2$ and from this formula (B) follows easily, $0<\alpha<2$. It is perhaps of interest to note that if $\alpha=2$, formula (B) reduces to

$$
E_{x}\left(T^{2}\right)=\frac{r^{2}}{2 N^{2}}\left(r^{2}-|x|^{2}\right)-\frac{1}{4 N(N+2)}\left(r^{4}-|x|^{4}\right)
$$

and this is easily seen to be correct, even though the computation leading to formula (B) is only valid for $0<\alpha<2$.

Note added in revision. Since the first version of this paper was written, $\mathrm{H}$. Widom, Stable processes and integral equations, Trans. Amer. Math. Soc. vol. 98 (1961) pp. 430-450, has computed the potential kernel for the absorb- 
ing barrier process on $G=\{x:|x|<1\}$ in the case $N=1$. That is, he found $\int_{0}^{\infty} k(t, x, y) d t$ and so $E_{x}(T)$ could be computed by integrating his result over $G$. Also all the moments of $T$ may be expressed in terms of the potential kernel, and so in essence he has all the moments of $T$.

Appendix. Proof of Theorem 6.2. Throughout this section our standard reference for properties of hypergeometric functions will be [11]. Also $M$ will denote a positive constant, not necessarily the same at each appearance.

LEMMA 1. The function $q$ defined in Theorem 6.2 is in $L_{1}\left(R^{N}\right)$.

Proof. It is simple to see that $q$ is integrable except possibly at $|x|=1$. For $1<|x| \leqq 2$ we have

$$
\begin{aligned}
|q(x)| & \leqq M \int_{0}^{1} t^{-1 / 2}\left(1-t|x|^{2}\right)^{-1-\alpha / 2} d t \\
& \leqq M F\left(1+\frac{\alpha}{2}, \frac{1}{2} ; \frac{3}{2} ;|x|^{-2}\right) \\
& \leqq M\left(|x|^{2}-1\right)^{-\alpha / 2}
\end{aligned}
$$

and thus $q$ is integrable near $|x|=1$.

LEMMA 2.

$$
\begin{aligned}
& {\left[\alpha K(\alpha, N)^{2} \pi^{N / 2} 2^{(N+\alpha) / 2}\right]^{-1} h(\xi)} \\
& =\Gamma\left(\frac{N+\alpha}{2}\right) \Gamma(\alpha)\left[\Gamma\left(\frac{N}{2}+\alpha\right)|\xi|^{(N+\alpha) / 2}\right]^{-1} J_{(N+\alpha) / 2}(|\xi|) \\
& \quad+\frac{N \alpha}{N+\alpha} \Gamma\left(\frac{\alpha}{2}\right)|\xi|^{-(N+\alpha) / 2} \\
& \quad \quad \cdot \int_{0}^{1} t^{(N+\alpha+2) / 2} J_{(N+\alpha) / 2}(t|\xi|) F\left(\frac{2-\alpha}{2}, \frac{N+2}{2} ; \frac{N+\alpha+2}{2} ; t^{2}\right) d t .
\end{aligned}
$$

Proof. We write

$$
h(x)=2 \alpha K(\alpha, N)^{2} \int_{0}^{1} \operatorname{th}(t, x) F\left(-\frac{\alpha}{2}, \frac{N}{2} ; \frac{N+\alpha}{2} ; t^{2}\right) d t
$$

where

$$
h(t, x)=\left\{\begin{array}{cl}
\left(t^{2}-|x|^{2}\right)^{\alpha / 2-1}, & t \geqq|x|, \\
0, & t<|x| .
\end{array}\right.
$$

From formula $33, \S 8.5$ of [5] we have

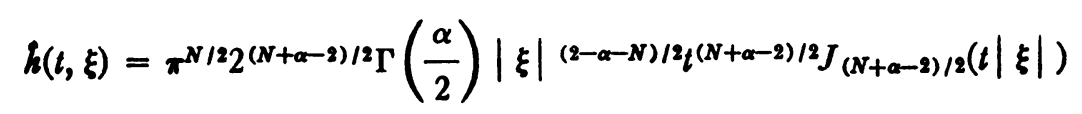


and it is easy to see that in computing $h(\xi)$ we can interchange the order of integration. Computing $h(\xi)$ in this manner and then integrating by parts (we differentiate the term $\left.F\left(-\alpha / 2, N / 2 ;(N+\alpha) / 2 ; t^{2}\right)\right)$ we obtain Lemma 2.

LEMMA 3. If $|x|<1$, then $\left(1-|x|^{2}\right)^{\alpha / 2}=\phi(x)+\psi(x)$ where

$$
\phi(x)=F\left(-\frac{\alpha}{2}, \frac{N}{2} ; \frac{N+\alpha}{2} ;|x|^{2}\right)
$$

and

$$
\begin{aligned}
\psi(x)= & \frac{2 N \alpha}{(N+\alpha)^{2}} \Gamma\left(\frac{N}{2}\right)\left[\Gamma\left(\frac{N+\alpha}{2}\right) \Gamma\left(-\frac{\alpha}{2}\right)|x|^{N+\alpha}\right]^{-1} \\
& \times \int_{0}^{|x|} t^{N+\alpha+1} F\left(\frac{N+\alpha}{2}, \frac{2+\alpha}{2} ; \frac{N+\alpha+2}{2} ; t^{2}|x|^{-2}\right) \\
& \cdot F\left(\frac{2-\alpha}{2}, \frac{N+2}{2} ; \frac{N+\alpha+2}{2} ; t^{2}\right) d t .
\end{aligned}
$$

Of course, $\psi(0)=\lim _{x \rightarrow 0} \psi(x)=0$.

Proof. This is clear if $x=0$. Let $x$ with $0<|x|<1$ and $\alpha$ with $0<\alpha<2$ be fixed. If we regard $\phi(x)+\psi(x)$ as a function of $N$ for complex values of $N$, it is not difficult to see that $\phi+\psi$ is analytic in $N$ provided $\operatorname{Re}(N)>0$. Perhaps the simplest way to see this is to use the integral representation (p. 8 of [11]) for the hypergeometric function and then apply Morera's theorem. Thus using analytic continuation it will suffice to prove Lemma 3 for real $\boldsymbol{N}$ satisfying $N>2-\alpha$.

In the expression for $\psi(x)$ we first make the change of variable $u=t^{2}$ and then integrate by parts twice, the first time integrating the term, $F((N+\alpha) / 2,(2+\alpha) / 2 ;(N+\alpha+2) / 2 ; u)$, the second time integrating the term $F\left((2-\alpha) / 2,(N+2) / 2 ;(N+\alpha) / 2 ; u|x|^{2}\right)$. In this way we find (in the second integration by parts we need $N>2-\alpha)$

$$
\begin{aligned}
& \psi(x)=\frac{-N \alpha|x|^{2}}{(N+\alpha)(N+\alpha-2)} F\left(\frac{2-\alpha}{2}, \frac{N+2}{2} ; \frac{N+\alpha+2}{2} ;|x|^{2}\right) \\
& -F\left(-\frac{\alpha}{2}, \frac{N}{2} ; \frac{N+\alpha-2}{2} ;|x|^{2}\right) \\
& +\frac{(N+\alpha-2) \Gamma\left(\frac{N}{2}\right)}{2 \Gamma\left(1-\frac{\alpha}{2}\right) \Gamma\left(\frac{N+\alpha}{2}\right)} \\
& \int_{0}^{1} t^{(N+\alpha+2) / 2}(1-t)^{-\alpha / 2} F\left(-\frac{\alpha}{2}, \frac{N}{2} ; \frac{N+\alpha-2}{2}, t|x|^{2}\right) d t .
\end{aligned}
$$


Calling this last integral $J$ and using the integral representation on p. 11 of [11] we have

$$
J=\Gamma\left(\frac{N+\alpha-2}{2}\right) \Gamma\left(1-\frac{\alpha}{2}\right)\left[\Gamma\left(\frac{N}{2}\right)\right]^{-1}\left(1-|x|^{2}\right)^{\alpha / 2} .
$$

Combining all these results and using the third recursion formula on p. 9 of [11] we obtain Lemma 3.

LEMMA 4. The function $\phi$ defined in Lemma 3 can also be written

$$
\begin{aligned}
\phi(x)= & \Gamma(\alpha) \Gamma\left(\frac{N+\alpha}{2}\right)\left[\Gamma\left(\frac{N}{2}+\alpha\right) \Gamma\left(\frac{\alpha}{2}\right)\right]^{-1} \\
& +\frac{N \alpha}{N+\alpha} \int_{|x|}^{1} t F\left(\frac{2-\alpha}{2}, \frac{N+2}{2} ; \frac{N+\alpha+2}{2} ; t^{2}\right) d t .
\end{aligned}
$$

Proof. This is an immediate consequence of the second differentiation formula of p. 10 of [11].

LEMMA 5. If $|x|>1$, then

$$
\begin{aligned}
& q(x)=\frac{4}{N+\alpha} K(\alpha, N) \Gamma\left(\frac{N}{2}\right) \Gamma(\alpha)\left[\Gamma\left(-\frac{\alpha}{2}\right) \Gamma\left(\frac{\alpha}{2}\right) \Gamma\left(\frac{N}{2}+\alpha\right)|x|^{N+\alpha}\right]^{-1} \\
& \times F\left(\frac{N+\alpha}{2}, \frac{2+\alpha}{2} ; \frac{N+\alpha+2}{2} ;|x|^{-2}\right) \\
&+\frac{2 N \alpha}{(N+\alpha)^{2}} K(\alpha, N) \Gamma\left(\frac{N}{2}\right)\left[\Gamma\left(-\frac{\alpha}{2}\right) \Gamma\left(\frac{N+\alpha}{2}\right)|x|^{N+\alpha}\right]^{-1} \\
& \times \int_{0}^{1} t^{(N+\alpha) / 2} F\left(\frac{2-\alpha}{2}, \frac{N+2}{2} ; \frac{N+\alpha+2}{2} ; t\right) \\
& \times F\left(\frac{N+\alpha}{2}, \frac{2+\alpha}{2} ; \frac{N+\alpha+2}{2} ; t|x|^{-2}\right) d t .
\end{aligned}
$$

Proof. This follows easily if we integrate by parts.

Let $H(x)$ be the function defined in (5.2) and set $H(t, x)=t^{-(N+\alpha) / 2} H(x / t)$ if $t>0$. If we combine Lemmas 3,4 , and 5 we can write

$$
\begin{aligned}
q(x)=2 K(\alpha, N) \Gamma\left(\frac{N+\alpha}{2}\right) \Gamma(\alpha)\left[\Gamma\left(\frac{N}{2}+\alpha\right) \Gamma\left(\frac{\alpha}{2}\right)\right]^{-1} H(1, x) \\
+\frac{2 N \alpha}{N+\alpha} K(\alpha, N) \\
\quad \times \int_{0}^{1} t^{(N+\alpha+2) / 2} H(t, x) F\left(\frac{2-\alpha}{2}, \frac{N+2}{2} ; \frac{N+\alpha+2}{2} ; t^{2}\right) d t .
\end{aligned}
$$


Also Theorem 5.2 implies that

(A-2) $\quad \hat{H}(t, \xi)=(2 \pi)^{N / 2} \Gamma\left(\frac{N}{2}\right)\left[2^{\alpha / 2} \Gamma\left(\frac{N+\alpha}{2}\right)\right]^{-1}|\xi|^{(\alpha-N) / 2 J_{(N+\alpha) / 2}(t|\xi|) .}$

From (A-1) and (A-2) we can compute $q$. The necessary interchange in order of integration is easily justified. Combining this result with Lemma 2 we see that $\hat{q}(\xi)=|\xi| \alpha h(\xi)$. This completes the proof of Theorem 6.2.

\section{REFERENCES}

1. R. M. Blumenthal and R. K. Getoor, Some theorems on stable processes, Trans. Amer. Math. Soc. vol. 95 (1960) pp. 263-273.

2. -The asymptotic distribution of the eigenvalues for a class of Markov operators, Pacific J. Math. vol. 9 (1959) pp. 399-408.

3. S. Bochner and K. Chrandrasekharan, Fourier transforms, Annals of Mathematics Studies, no. 19, Princeton University Press, 1949.

4. J. Elliot, Absorbing barrier processes connected with the symmetric stable densities, Illinois J. Math. vol. 3 (1959) pp. 200-216.

5. A. Erdélyi, Tables of integral transforms, Vol. II, Bateman Manuscript Project, New York, McGraw-Hill, 1954.

6. R. K. Getoor, Additive functionals of a Markoo process, Pacific J. Math. vol. 7 (1957) pp. 1577-1591.

7. - Markov operators and their associated semi-groups, Pacific J. Math. vol. 9 (1959) pp. 449-472.

8. E. Hille and R. S. Phillips, Functional analysis and semi-groups, Amer. Math. Soc. Colloquium Publications, vol. 31, 1957.

9. M. Kac, Some remarks on stable processes with independent increments, Probability and Statistics, The Harald Cramér Volume, New York, Wiley, 1960.

10. M. Kac and H. Pollard, Partial sums of independent random variables, Canad. J. Math vol. 11 (1950) pp. 375-384.

11. W. Magnus and F. Oberhettinger, Formulas and theorems for the special functions of mathematical physics, New York, Chelsea, 1949.

12. D. Ray, Stable processes with an absorbing barrier, Trans. Amer. Math. Soc. vol. 39 (1958) pp. 16-24.

13. E. C. Titchmarsh, Introduction to the theory of Fourier integrals, Oxford, Clarendon Press, 1948.

UNIVERSITY OF WASHINGTON,

Seatthe, Washington 